\title{
Erratum to: Thermodynamic Simulation for Interaction of Polychlorinated Biphenyls with Potassium Hydroxide in Polyalkanolamines
}

\author{
A. V. Maiorova ${ }^{a}$,* T. V. Kulikova $a$, T. I. Gorbunova ${ }^{b}$, and M. G. Pervova ${ }^{b}$ \\ a Institute of Metallurgy, Ural Branch, Russian Academy of Sciences, Yekaterinburg, 620016 Russia \\ ${ }^{b}$ Postovsky Institute of Organic Synthesis, Ural Branch, Russian Academy of Sciences, Yekaterinburg, 620137 Russia \\ *e-mail:imeturoran@mail.ru
}

Received May 5, 2021; Received May 5, 2021; Received May 5, 2021

DOI: $10.1134 / \mathrm{S} 1070427221040169$

Author affiliation mark at T.I. Gorbunova should be replaced by "b".

Author affiliation mark at M.G. Pervova should be replaced by "b".

The original article can be found online at https://doi.org/10.1134/S1070427221030095 